\title{
Mid-Infrared Reflect-Array Antenna With Beam Switching Enabled by Continuous Graphene Layer
}

\author{
Henry Giddens ${ }^{\circledR}$, Liang Yang, Jing Tian, and Yang Hao, Fellow, IEEE
}

\begin{abstract}
Tunable graphene conductivity can enable dynamic control of the reflection characteristics of near-resonant electromagnetic structures. In this letter, a mid-infrared reflectarray antenna with binary beam switching is presented. The proposed structure consists of a uniform graphene monolayer patterned with an aperiodic array of near-resonant metallic split-ring resonators. By controlling the chemical potential of the continuous graphene layer through electrostatic biasing, the reflection phase and amplitude of the split-ring resonator is controlled, providing binary beam switching capability to the reflect-array.
\end{abstract}

Index Terms-Graphene, mid-infrared, THz, reflect-array antenna.

\section{INTRODUCTION}

$\mathbf{I}$ $\mathrm{N}$ RECENT years, the mid-infrared (IR) and $\mathrm{THz}$ frequency bands have gained the interest of a number of industries for applications such as wireless communications, imaging and remote sensing [1], [2]. Many of these require the system to be reconfigurable for wave radiation and reception so as to increase communication capacity and minimize the interference from other sources. One way to achieve beam steering of electromagnetic (EM) radiation is through a reflective surface with a spatial phase distribution, otherwise known as a reflect-array antenna. These devices have been investigated by the research community for applications at frequencies spanning microwaves to optics [3]-[5]. Ideally, the unit-cell elements of a reflect-array antenna should be capable to cover a full phase-variation region of $360^{\circ}$. However, at infrared (IR) spectrum, loss effects of metal and dielectric can restrict the phase variation to a narrow range in some cases [6]. By using low-loss materials at IR spectrum, the design of gold square-patch elements has been able to obtain $292^{\circ}$ phase shift at $28.3 \mathrm{THz}$ [7]. Further, high reflection efficiency of $92 \%$ has been achieved [8]. However, the phase variation

Manuscript received September 26, 2017; revised January 11, 2018; accepted March 5, 2018. Date of publication March 12, 2018; date of current version March 27, 2018. This work was supported in part by the Engineering and Physical Sciences Research Council under Grant EP/K01711X/1, in part by the EU Graphene Flagship under Grant FP7-ICT-604391, in part by Graphene Core 1 under Grant H2020 696656, and in part by the China Scholarship Council. (Henry Giddens and Liang Yang contributed equally to this work.) (Corresponding author: Henry Giddens.)

The authors are with the School of Electronic Engineering and Computer Science, Queen Mary University of London, London E1 4NS, U.K. (e-mail: h.giddens@qmul.ac.uk; y.hao@qmul.ac.uk).

Color versions of one or more of the figures in this letter are available online at http://ieeexplore.ieee.org.

Digital Object Identifier 10.1109/LPT.2018.2814684 of these designs depends on the physical dimensions of the patch elements, which are made from conventional metals and cannot be dynamically tuned after fabrication.

The 2D material graphene, possesses a chemical-potentialdependent complex conductivity which can be tuned under electrostatic bias [9], [10]. This unique property has motivated researchers to investigate various graphene-based applications [11]. Moreover, at mid-IR spectrum, graphene exhibits low-loss plasmonic-like complex surface conductivity [12], which provides great potential on novel tunable mid-IR reflectarray antennas. A design of reflect-array using gate-controlled aperiodic graphene nanoribbon has been proposed [13]. The nano-scaled graphene meta-surface requires a large number of graphene nano-ribbon resonators with a spatially varying width profile. By tuning the chemical potential of graphene resonators, the reflect-array is able to steer an incident light beam towards different directions. However, this design requires nano-scale patterning of a large number of graphene nano-ribbons, which is more difficult to achieve in practice compared with a single continuous graphene sheet. With the fabrication advantage of continuous graphene, researchers have proposed to directly load unit cells of gold rod antennas [14]. However, these designs are more suited for amplitude and phase modulation of the reflected light uniformly across the reflect-array, rather than beam steering through a phasegradient surface.

In this letter, a graphene enabled reflect-array antenna is presented. The phase gradient of the EM surface is achieved by an aperiodic array of split-ring resonator (SRR) structures. The novelty of this design lies in the utilization of a continuous single graphene sheet on which the SRR structures are deposited. The simplicity of the design and size of the features are suitable for applying common fabrication techniques such as chemical vapour disposition (CVD) and electron beam photolithography. By tuning the chemical potential of graphene sheet through electrostatic biasing, binary-state beam switching of the far-field radiation is obtained.

\section{Unit Cell Design}

An electromagnetic surface with a reconfigurable phase gradient is able to steer reflected light in multiple directions. A planar array of near-resonant electromagnetic structures can be engineered to reflect an incoming beam of light to a given angle according to the Snell's Law [5]. Using the technique of array synthesis, the desired phase-gradient between adjacent 


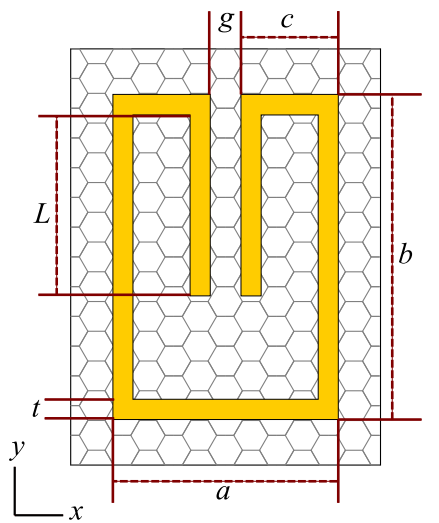

(a)

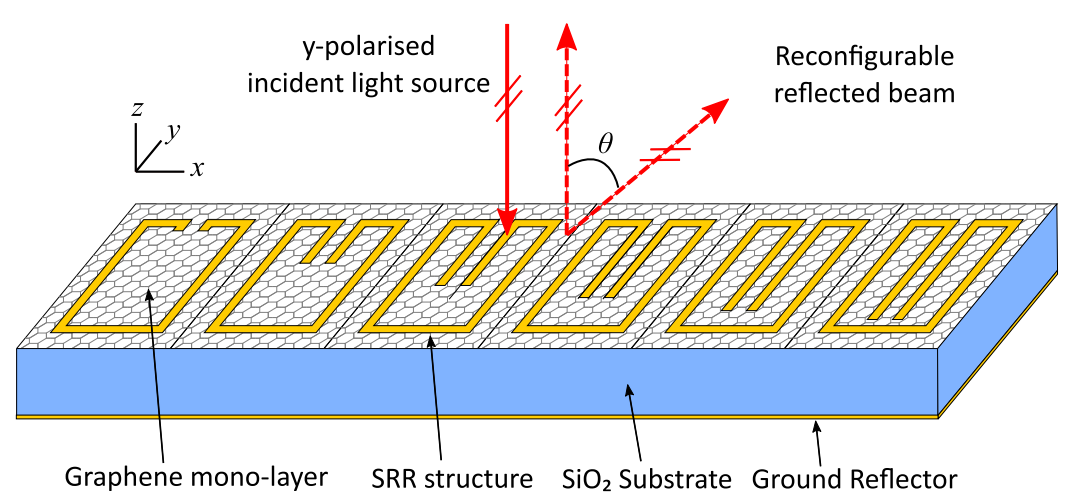

(b)

Fig. 1. (a) Schematic of the reconfigurable graphene reflect-array unit cell. (b) Diagram of the 6-element super unit cell showing the graphene monolayer, SRR structures, substrate and reflector.

array elements can be calculated through (1).

$$
\frac{d \phi}{d x}=\sin \theta \times \frac{2 \pi}{\lambda}
$$

where $d \phi$ represents the phase difference between elements, $\mathrm{dx}$ is the dimension of a single element in the $\mathrm{x}$-direction, and $\theta$ is the angle of the reflected beam of light with respect to the incident wave. By utilizing tunable conductivity properties of graphene, a reconfigurable phase gradient surface has been designed where the direction of the reflected light beam can be controlled.

The unit cell of the proposed antenna array consists of a near-resonant split-ring metallic structure deposited onto a continuous layer of graphene. These structures have been previously used to design a phase-gradient meta-surface as a spoof plasmon polariton coupler in the microwave range [15], and provide a simple means of controlling the reflection phase when illuminated with an incident light source. In order to obtain a constant phase gradient across the surface of the reflect-array, the inner tooth length of the split-ring is varied (Fig. 1 (b)). By changing the chemical potential of the graphene layer, the reflection phase of the unit cell can be modified. By carefully selecting the inner tooth length of each unit cell at the desired frequency of operation, the phase gradient of the whole surface can be switched between two distinct states by tuning the graphene chemical potential. As a result, a binary beam-steerable reflect-array enabled by a continuous graphene monolayer can be realised.

Fig. 1 (a) shows the structure of the unit cell, which consists of a continuous graphene sheet deposited onto a $\mathrm{SiO}_{2}$ substrate with $\varepsilon_{r}=3$ at the relevant wavelengths [16], [17], which is backed by a metallic ground plane. A split-ring resonator structure is patterned onto the graphene layer, and the inner tooth length $(\mathrm{L})$ is varied across the $\mathrm{x}$-dimension of the array. The unit cell has $\mathrm{x}-\mathrm{y}$ dimensions of $2 \times 3 \mu \mathrm{m}^{2}$, and a thickness of $0.5 \mu \mathrm{m}$. The dimensions of the split-ring structure were as follows, $a=1.6 \mu \mathrm{m}, b=2.4 \mu \mathrm{m}, c=0.76 \mu \mathrm{m}$, $g=0.08 \mu \mathrm{m}$ and $t=0.1 \mu \mathrm{m}$. The design dimensions of the SRR structure are well within the limitations of modern photolithographic techniques [16]. Simulations were performed using the commercial solver CST Microwave Studio in order to obtain the reflection amplitude and phase of the unit cell
TABLE I

Physical Graphene Properties Used in the Numerical Model

\begin{tabular}{|c|c|c|c|}
\hline$\mu_{c}(\mathrm{eV})$ & 0.1 & 0.5 & 0.9 \\
\hline$\tau_{s}(\mathrm{ps})$ & 0.1 & 0.1 & 0.1 \\
\hline $\begin{array}{c}\text { Electron mobility } \\
\left(\mathrm{cm}^{2} /(\mathrm{Vs})\right)\end{array}$ & $1 \times 10^{4}$ & $2 \times 10^{3}$ & $1.1 \times 10^{3}$ \\
\hline$Z_{s}(\Omega / s q)$ & $\begin{array}{c}16241+ \\
27663 \mathrm{i}\end{array}$ & $176+3477 \mathrm{i}$ & $95+1908 \mathrm{i}$ \\
\hline
\end{tabular}

as a function of both the graphene chemical potential and tooth length at a frequency of $32 \mathrm{THz}$. The complex surface conductivity, $\sigma_{s}$, for a continuous graphene sheet is given by Falkovsky [10]. In the numerical simulations, the graphene layer is modelled as an infinitesimally thin sheet with surface impedance equivalent to

$$
Z_{s}(\omega)=\frac{1}{\sigma_{s}(\omega)}
$$

A typical relaxation time of $\tau_{s}=0.1 \mathrm{ps}$ is assumed, and the chemical potential, $\mu_{c}$ is varied between $0.1,0.5$ and $0.9 \mathrm{eV}$ to achieve the required phase gradient modification. Table I lists the calculated parameters of the graphene sheet for these 3 different states. Floquet boundary conditions were applied to account for mutual coupling between adjacent cells.

The reflection phase and amplitude of a y-polarised incident beam vs. the unit cell tooth length, L, are shown in Fig. 2 for various graphene chemical potential biases. When the graphene layer is biased with $\mu_{c}=0.1 \mathrm{eV}$, the reflection phase of the unit cell is strongly dependent on the value of L. As the tooth length is increased from $0.1 \mu \mathrm{m}$ to $2.1 \mu \mathrm{m}$ the reflection phase varies by almost $300^{\circ}$. The amplitude of the reflection coefficient varies between 0.95 and 0.6 , with the minimum at a tooth length of $1.6 \mu \mathrm{m}$, due to resonant effects of the metallic split-ring structure. When the $\mu_{c}$ is increased to $0.5 \mathrm{eV}$, the reflection phase is constant versus $\mathrm{L}$. The amplitude however varies significantly, between 0.8 and 0.25 . The resonant losses in the reflect-array antenna will ultimately affect its overall gain and the shape of its radiation pattern. Ideally, the magnitude of the reflection coefficient should be constant (and close to unity) otherwise the radiation pattern will have unwanted side lobes. When the $\mu_{c}$ is increased to 


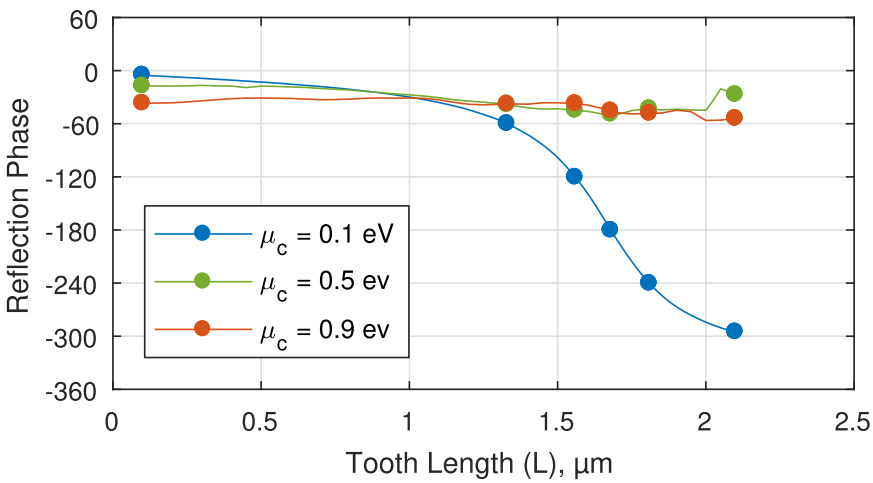

(a)

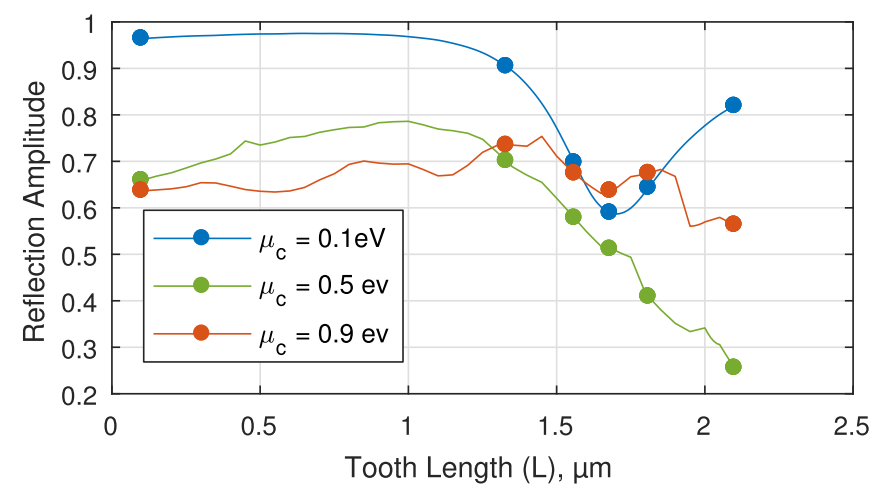

(b)

Fig. 2. Reflection Phase (a) and Amplitude (b) verses tooth length of the graphene enabled split-ring unit cell for various chemical potentials at a frequency of $32 \mathrm{THz}$. The markers indicate the discrete tooth lengths required to form the super unit cell that enables beam steering towards a $50^{\circ}$ angle when the $\mu_{c}$ of the graphene layer is $0.1 \mathrm{eV}$.

$0.9 \mathrm{eV}$, the reflection amplitude varies between 0.59 and 0.75 . The effects of the uneven amplitude and phase distribution on the radiation pattern and gain of the reflect-array are analysed further in Section IV. As can be seen from Table I, the surface impedance of the graphene sheet is significantly reduced when the chemical potential is increased from $0.1 \mathrm{eV}$. This has the effect of altering the impedance created by the capacitance between the inner-teeth of the SRR structure, shifting the resonant frequency away from the operational frequency of $32 \mathrm{THz}$ regardless of the dimension $L$. This allows us to design an array with two chemical potential dependent, constant, phase gradient profiles that are required in order achieve the binary beam switching effect.

From equation (1) it can be determined that, in order to reflect a $32 \mathrm{THz}$ incident wave to an angle of $50^{\circ}$, a phase shift $60^{\circ}$ between adjacent elements on the reflective surface is required and this can be achieved with 6 adjacent unit cells. The curve markers on Fig. 2 indicate the tooth lengths of adjacent unit cells that are required to form the super unit cell. The super unit cell is shown in Fig. 1 (b).

\section{BEAM SWITCHING}

The far-field radiation patterns of an array of spatially distributed unit cell elements can be computed using the array theory approach [18]. The unit-cell far field pattern is calculated from the numerical simulations by considering

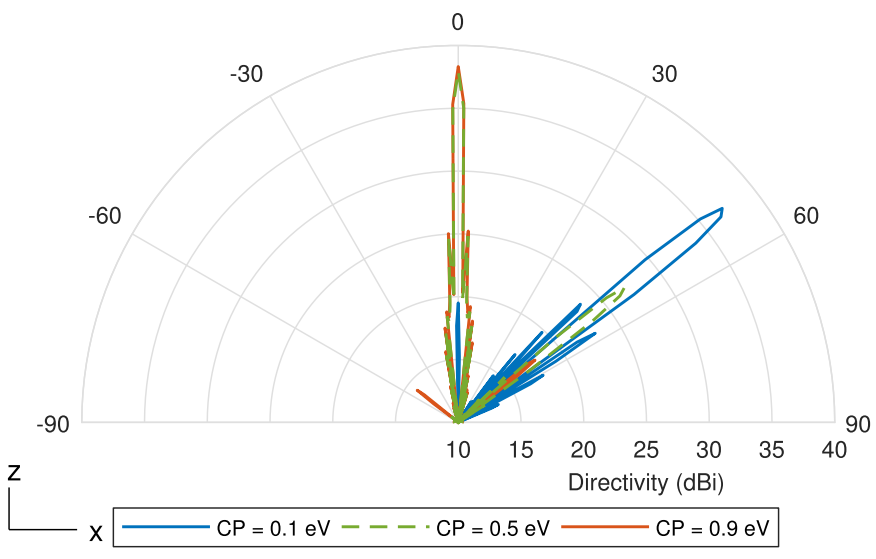

Fig. 3. Far field radiation pattern of $120 \times 80$ element graphene enabled reflect-array in $\mathrm{X}-\mathrm{Z}$ plane with the chemical potential of the graphene layer equal to $0.1 \mathrm{eV}, 0.5 \mathrm{eV}$ and $0.9 \mathrm{eV}$.

the scattered fields in all directions above the array surface. The total radiation pattern is computed for an array of $120 \times 80$ elements -120 super unit cells along the $\mathrm{X}$-axis, and 80 along the $\mathrm{Y}$-axis (in the Y-axis, there is no phase variation between adjacent cells), and assumes a uniform illumination of the reflect-array surface from the incident light source. The dimensions of the complete array are therefore equivalent to approximately $20 \lambda$ in both the $\mathrm{X}$ and $\mathrm{Y}$ dimensions at $32 \mathrm{THz}$ occupying a total area of $240 \times 240 \mu \mathrm{m}^{2}$. The amplitude and phase weighting functions obtained from Fig. 2 were applied along with the relevant spatial phase shift in order to calculate the overall radiation pattern. Fig. 3 shows the far field radiation patterns in the $\mathrm{z}-\mathrm{x}$ plane when $\mu_{c}=0.1,0.5$ and $0.9 \mathrm{eV}$. As can be seen, when $\mu_{c}$ is equal to $0.1 \mathrm{eV}$ the beam is reflected towards a $\theta=50^{\circ}$ angle, and is reflected to $0^{\circ}$ when the chemical potential is increased to $0.9 \mathrm{eV}$. The maximum gain of the radiation pattern is equal to $38 \mathrm{dBi}$ when the chemical potential is $0.9 \mathrm{eV}$ and the incident wave is reflected back towards $\theta=0^{\circ}$, and falls slightly to $37 \mathrm{dBi}$ when the beam is steered towards the $50^{\circ}$ angle. When the chemical potential is equal to $0.5 \mathrm{eV}$, the main beam is towards the $0^{\circ}$ direction, however there is a significant side-lobe at $50^{\circ}$ due to the largely unequal reflection amplitude, although this is $10 \mathrm{~dB}$ weaker than the beam at $50^{\circ}$ when the chemical potential is equal to $0.1 \mathrm{eV}$.

The super unit cell beam steering results were confirmed through the full wave numerical simulations. Fig.4 shows the reflected $\mathrm{E}_{\mathrm{y}}$ field from the array surface up to a distance of $20 \mu \mathrm{m}(\approx 2 \lambda)$ for the two previously discussed $\mu_{c}$ states, normalized to the maximum value which occurs when $\mu_{c}=0.1 \mathrm{eV}$. It can clearly be seen that the direction of the $\mathrm{E}_{\mathrm{y}}$ field switches when the chemical potential is switched. When the chemical potential is equal to $0.9 \mathrm{eV}$, the maximum normalized field strength is at about 0.65 , which is in good agreement with the reflection coefficient results obtained from the single unit cell simulations shown in Fig. 2.

\section{FURTHER DiscusSiON}

The proposed reconfigurable reflect-array structure does not have the 'ideal' reflection phase and amplitude characteristics 

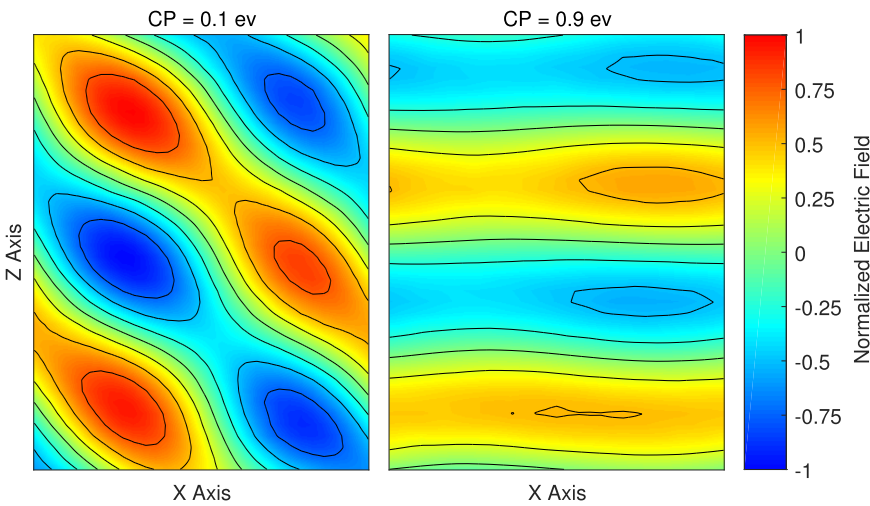

Fig. 4. $E_{y}$ field reflected from the array surface for graphene chemical potential of 0.1 and $0.9 \mathrm{eV}$. The bottom of the Z-axis is located immediately above the reflect-array surface.

that are typically required in optimal array designs. The initial array was designed with the intention that a constant phase gradient could be achieved across the surface for two different states, and that an even amplitude distribution could be achieved, ideally close to unity. Taking the average reflection coefficient for each of the two states, the overall losses (due to the resonance and substrate loss) are calculated to determine the antenna gain. When the chemical potential of the graphene layer is $0.1 \mathrm{eV}$, the reflection coefficient of the super unit cell is 0.77 , equating to losses of approximately $2.3 \mathrm{~dB}$. The maximum gain at an angle of $\theta=50^{\circ}$ is therefore equal to $34.7 \mathrm{~dB}$. When $\mu_{c}=0.9 \mathrm{eV}$, the average reflection coefficient amplitude is 0.65 and the losses $3.7 \mathrm{~dB}$ and the maximum gain of the reflected beam is $34.3 \mathrm{~dB}$. As a result of the higher reflection losses when $\mu_{c}=0.9 \mathrm{eV}$, the magnitude of the reflected light beam is approximately the same in both states. In practice, dynamically tuning between $0.1 \mathrm{eV}$ and $0.9 \mathrm{eV}$ would be difficult to achieve, requiring large electric fields. As demonstrated in Fig. 2 and Fig. 3, an incident light beam will also be reflected towards $0^{\circ}$ when the chemical potential is equal to $0.5 \mathrm{eV}$. However, the reflect-array exhibits larger amplitude losses with an average reflection amplitude of 0.5 and an associated maximum gain of $32 \mathrm{~dB}$. Furthermore, a side lobe appears towards the $50^{\circ}$ angle. In this state, the array does not perform optimally, however would still be useful in applications where the beam needs to be modulated between two different directions.

\section{CONCLUSION}

In this letter we have proposed a design for a grapheneenabled reconfigurable reflect-array surface operating in the mid-infrared spectrum. The proposed structure utilizes a continuous graphene sheet with an aperiodic array of near-resonant metallic split-ring structures. By tuning the chemical potential of the graphene sheet, we are able to switch the phase gradient of the reflective surface between two distinct states. The effect of an unequal reflection amplitude across the array surface have been considered, and light beam steering at two different states has been demonstrated. The proposed design opens up the possibility of novel applications at mid-infrared wavelength in imaging and communications.

\section{REFERENCES}

[1] D. C. Fernandez, R. Bhargava, S. M. Hewitt, and I. W. Levin, "Infrared spectroscopic imaging for histopathologic recognition," Nature Biotechnol., vol. 23, no. 4, p. 469-475, 2005.

[2] A. P. M. Michel et al., "Quantum cascade laser open-path system for remote sensing of trace gases in Beijing, China," Opt. Eng., vol. 49, no. 11, p. $111125,2010$.

[3] J. Huang and J. A. Encinar, Reflectarray Antennas. Hoboken, NJ, USA: Wiley, 2007.

[4] T. Niu et al., "Experimental demonstration of reflectarray antennas at terahertz frequencies," Opt. Exp., vol. 21, no. 3, pp. 2875-2889, 2013.

[5] N. Yu et al., "Light propagation with phase discontinuities: Generalized laws of reflection and refraction," Science, vol. 334, no. 6054, pp. 333-337, Oct. 2011.

[6] F. Yang et al., "Reflectarray design at infrared frequencies: Effects and models of material loss," IEEE Trans. Antennas Propag., vol. 60, no. 9, pp. 4202-4209, Sep. 2012.

[7] J. C. Ginn, B. A. Lail, and G. D. Boreman, "Phase characterization of reflectarray elements at infrared," IEEE Trans. Antennas Propag., vol. 55, no. 11, pp. 2989-2993, Nov. 2007.

[8] M. Farmahini-Farahani and H. Mosallaei, "Birefringent reflectarray metasurface for beam engineering in infrared," Opt. Lett., vol. 38, no. 4, pp. 462-464, 2013.

[9] V. W. Brar, M. S. Jang, M. Sherrott, J. J. Lopez, H. A. Atwater, and J. Thomas, "Highly confined tunable mid-infrared plasmonics in graphene nanoresonators," Nano Lett., vol. 13, no. 6, pp. 2541-2547, 2013.

[10] L. A. Falkovsky, "Optical properties of graphene," J. Phys., Conf. Ser., vol. 129, no. 1, p. 012004, 2008.

[11] T. Low and P. Avouris, "Graphene plasmonics for terahertz to midinfrared applications," ACS Nano, vol. 8, no. 2, pp. 1086-1101, 2014.

[12] S. H. Mousavi et al., "Inductive tuning of fano-resonant metasurfaces using plasmonic response of graphene in the mid-infrared," Nano Lett., vol. 13, no. 3, pp. 1111-1117, 2013.

[13] E. Carrasco, M. Tamagnone, J. R. Mosig, T. Low, and J. Perruisseau-Carrier, "Gate-controlled mid-infrared light bending with aperiodic graphene nanoribbons array," Nanotechnology, vol. 26, no. 13, p. 134002, 2015.

[14] Z. Li and N. Yu, "Modulation of mid-infrared light using graphene-metal plasmonic antennas," Appl. Phys. Lett., vol. 102, no. 13, p. 131108, 2013.

[15] J. Wang et al., "High-efficiency spoof plasmon polariton coupler mediated by gradient metasurfaces," Appl. Phys. Lett., vol. 101, no. 20, p. 201104, 2012.

[16] E. Buitrago, R. Fallica, D. Fan, T. S. Kulmala, M. Vockenhuber, and Y. Ekinci, "SnO $x$ high-efficiency EUV interference lithography gratings towards the ultimate resolution in photolithography," Microelectron. Eng., vol. 155, pp. 44-49, Apr. 2016.

[17] G. Power, J. K. Vij, and M. Shaw, "Refractive index at infrared wavelengths and dielectric permittivity of pure and fluorinated silicon dioxide from measurements of their thin films deposited on $\mathrm{Si}$," J. Phys. D, Appl. Phys., vol. 37, no. 9, p. 1362, 2004.

[18] C. A. Balanis, Antenna Theory: Analysis and Design. Hoboken, NJ, USA: Wiley, 2005.

[19] S. V. Hum and J. Perruisseau-Carrier, "Reconfigurable reflectarrays and array lenses for dynamic antenna beam control: A review," IEEE Trans. Antennas Propag., vol. 62, no. 1, pp. 183-198, Jan. 2014.

[20] K.-S. Lee, T.-M. Lu, and X. C. Zhang, "Tera tool [terahertz time-domain spectroscopy]," IEEE Circuits Devices Mag., vol. 18, no. 6, pp. 23-28, Nov. 2002. 